\title{
ESTIMATING AND UNDERSTANDING VERTICAL STRUCTURE OF FORESTS FROM MULTIBASELINE TANDEM-X POL-INSAR DATA
}

\author{
M. Pardini, A. Toraño-Caicoya, F. Kugler, K. Papathanassiou \\ German Aerospace Center (DLR), Microwave and Radar Institute \\ Oberpfaffenhofen, Germany
}

\begin{abstract}
TanDEM-X (TDX) forms with TerraSAR-X (TSX) the first single-pass synthetic aperture radar (SAR) interferometer in space with polarimetric capabilities. The availability of such system allows for the first time the acquisition and analysis of X-band Pol-InSAR data from space without the disturbing effect of temporal decorrelation. After two years of mission, time series with variable baseline over the same forest sites are available, allowing to (1) explore their information content, (2) assess penetration capabilities, (3) assess scattering model assumptions, and (4) estimate vertical structure and monitor its dynamics.

This paper discusses for the first time the potential of estimating forest vertical structure from spaceborne single-pass interferometers, extending classical tomographic concepts. Results of first experiments with TSX/TDX multibaseline Pol-InSAR data acquired over the Tapajos national forest (Brazil) are shown. Especially regarding tropical forests, potentials and applications of X-band for forest structure monitoring will also be discussed.
\end{abstract}

Index Terms - Synthetic Aperture Radar (SAR), polarimetry, coherence tomography, parameter inversion, spectral estimation, forest structure.

\section{INTRODUCTION}

In the recent past, space borne repeat-pass implementations of forest vertical structure monitoring have been hampered by the inherent presence of short/long term temporal decorrelation. Mainly for this reason, polarimetric space borne missions as the RadarSAT-2 (C-band) or ALOS-PalSAR (Lband) could not contribute essential neither with respect to large scale demonstration of Pol-InSAR techniques nor for the development of new Pol-InSAR application areas [1]. The acquisition and analysis of Pol-InSAR data without the disturbing effect of temporal decorrelation on a global scale is possible since 2010 by means of the X-band TSX-TDX mission, which provides for the first time single pass (singleand dual-) polarimetric interferometric data from space [2]. Recent TDX experiments for forest applications have demonstrated rather surprising penetration capabilities of X-band radar signals into volumes, primarily for boreal and less dense forest environments. Different scattering behaviours have been observed for different seasons, for instance between summer and winter in the case of boreal forests and between wet season and dry season in the case of tropical forest systems [1]. Pol-InSAR forest height inversion has been demonstrated to be possible using either dual pol acquisitions or a single pol acquisition in combination with an external terrain DEM [1]. Combining forest height and allometric equations, the possibility has also been shown of generating high resolution biomass classification maps of boreal forests improving previously available thematic maps [3].

After two years of missions, time series with variable baseline over the same forest sites are available. The objective of this paper is to evaluate the tomographic capabilities offered by a set of tandem acquisitions with variable baseline, and to understand the potential for 3-D forest monitoring at $\mathrm{X}$-band. The methodology for estimating the vertical structure from a coherence vector is presented, and first results over the Tapajos national forest (Brazil) are shown. In addition, potential and applications of X-band for forest structure monitoring will be discussed.

\section{COHERENCE TOMOGRAPHY FROM SPACE}

Let $\gamma$ be a $K$-dimensional vector collecting $K$ temporal decorrelation-free coherences $\gamma_{k}, k=1, \ldots, K$, corresponding to the different vertical wavenumbers $k_{z, k}$ at each pass of the TSX-TDX couple. Differently from the classical 3-D SAR tomography, in which the vertical backscattering profile and parameters are estimated by combining the SLC data, in this case $\gamma$ constitute the only observable of the estimation problem. As a consequence, the estimation of the vertical profile can then be recast in the general framework of coherence tomography [4].

Considering for a while that the $k_{z, k}$ are uniformly distributed, the conventional approach to estimate the power spectrum from $\gamma$ is to assume $\gamma_{k}=0$ for $k>K$ and to simply take the Fourier transform of $\gamma$. However, problems of sidelobes and resolution in height generally arise, reducing the quality of the spectral estimate. 
In order to obtain profile estimates with higher quality, the vertical structure could be either extracted by means of a model based inversion from the interferometric coherences, or by approximating the structure function through a weighted sum of a series of basis functions [4]. This last approach, however, requires an a priori information about the two heights limiting the volume, and is not suitable for imaging non-volumetric scatterers.

In the following, two approaches will be proposed. The first one can be regarded as a multibaseline (MB) complex coherence combination for estimating a high resolution DEM. The second one constitutes a way to estimate the vertical profile of the backscattered power without the need of any a priori information.

\subsection{Accurate DEM estimation}

It is well known from the InSAR literature that MB InSAR can be successfully exploited for automatic phase unwrapping and high resolution DEM reconstruction. The resulting accuracy in height estimation improves at the increase of the number of looks and the number of coherences. As a consequence, fixed a desired accuracy, one can exploit the degree of freedom on the number of baselines to reduce the width of the multilook cell, thus increasing the horizontal resolution of the final product. A high resolution DEM could find an important application in the identification and positioning of large trees in tropical forests. Indeed, large trees are particularly significant for the carbon regime, they contain around the $50 \%$ of biomass, and they influence strongly the structure of the forest around them [5]. The higher the resolution, the better the identification.

Height inversion can be improved by accounting for a decorrelation pattern along the baselines. A very simple model is the following:

$$
\gamma_{k}=\rho\left(k_{z, k}\right) e^{j k_{z, k} z_{D}},
$$

$k=0, \ldots, K$, where $z_{D}$ is the DEM height to be estimated and $\rho\left(k_{z, k}\right)$ is a real-valued decorrelation pattern. By using a coherence fitting criterion, the estimators of $z_{D}$ and $\rho\left(k_{z, k}\right)$ are decoupled and can be written as:

$$
\begin{gathered}
\hat{z_{D}}=\arg \min _{z_{D}} \sum_{k=1}^{K} \Re^{2}\left\{-j \gamma_{k} e^{-j k_{z, k} z_{D}}\right\}, \\
\hat{\rho}\left(k_{z, k}\right)=\Re\left\{\gamma_{k} e^{-j k_{z, k} \hat{z}_{D}}\right\} .
\end{gathered}
$$

It is worth noting that the decorrelation $\hat{\rho}\left(k_{z, k}\right)$ may be used to infer penetration and forest structure properties. The model (1) can be modified to include the response of more than one decorrelating scatterers at different heights. In this case, scatterers in layover can be solved, increasing the accuracy and reducing the bias of the DEM of the top of the canopy.

\subsection{Vertical structure estimation}

The model-based algorithm of Section 2.1 only estimates the parameters characterizing the vertical structure, and from these parameters a vertical backscattering profile can be directly calculated. Concerning non-model based approaches, a high-resolution full vertical structure in the classical tomographic SAR sense could be estimated if a coherence matrix $\Gamma$ instead of the coherence vector $\gamma$ would be available. A simple solution consists in interpolating $\gamma$ to obtain a "virtual" vector $\gamma_{I}$ corresponding to uniformly spaced baselines using the method proposed in [6]. Remembering that a coherence matrix of a uniformly spaced baseline distribution has a Toeplitz structure, the whole matrix is defined only by its first row. As a consequence, a matrix $\boldsymbol{\Gamma}_{I}$ could be filled starting from $\gamma_{I}$. However, such a solution is not guaranteed to be positive definite, and no reasonable inversion is possible.

A way to circumvent this shortcoming is to make use of different polarization channels, and to assume valid the random volume hypothesis [1]. In this case, the MB PolInSAR coherence matrix $\mathbf{W}$ can be expressed as a sum of Kronecker products, each one corresponding to a scattering mechanism (SM) [7]. To obtain an estimate of $\mathbf{W}$, we propose the following procedure:

- calculate the polarimetric-interferometric coherences at each baseline, obtaining the vectors $\gamma_{A B}$ where $A, B \in$ $\{H H, V V, H V\}$;

- for each polarization combination, interpolate $\gamma_{A B}$ to $\gamma_{I, A B}$ by using the method in [6];

- fill a Toeplitz matrix $\boldsymbol{\Gamma}_{I, A B}$ for each $\gamma_{I, A B}$;

- fill the MB PolInSAR coherence matrix $\mathbf{W}$ by reordering the blocks $\boldsymbol{\Gamma}_{I, A B}$ into it.

Under the random volume hypothesis, $\mathbf{W}$ can be modelled as [7]:

$$
\mathbf{W}=\sum_{n=1}^{N_{S}} \mathbf{C}_{n} \otimes \mathbf{R}_{n},
$$

where $N_{S}$ is the number of SMs that compose the coherences, and $\mathbf{C}_{n}$ and $\mathbf{R}_{n}$ are the polarimetric and the structure coherence matrices, respectively, that can be estimated by means of an algebraic synthesis as explained in [7]. Limiting to $N_{S}=2 \mathrm{SMs}$, the structure-related coherence matrices are calculated as:

$$
\begin{aligned}
& \mathbf{R}_{1}=a \tilde{\mathbf{R}}_{1}+(1-a) \tilde{\mathbf{R}}_{2}, \\
& \mathbf{R}_{2}=b \tilde{\mathbf{R}}_{1}+(1-b) \tilde{\mathbf{R}}_{2},
\end{aligned}
$$

where $\tilde{\mathbf{R}}_{1}$ and $\tilde{\mathbf{R}}_{2}$ are estimated from the singular value decomposition of a permuted version of $\mathbf{W}$, and the constants $a$ and $b$ belong to an interval in which $\mathbf{R}_{1}$ and $\mathbf{R}_{2}$ are positive semidefinite. 


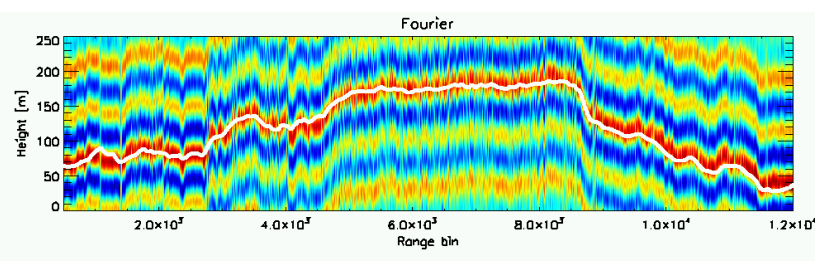

Fig. 1. Fourier tomographic slice in the slant range-height plane at fixed azimuth. The white line indicates the SRTM DEM.

\section{FIRST RESULTS}

\subsection{Test site and dataset}

First results have been obtained by processing a MB time serie of TSX/TDX data over the Amazonian Tapajos national forest, and they are presented in this Section. The Tapajos test site is located in the Amazon basin in northern Brazil beside the Tapajos River. The region of Tapajos is fairly flat and predominantly vegetated by undisturbed primary rainforest.

Five TSX/TDX dual-pol (HH/VV) interferograms were available for processing. They had been acquired between July and November 2011, i.e. during the dry season, with $k_{z}$ ranging almost uniformly from 0.04 to 0.1 and incidence angle of $40 \mathrm{deg}$. It has been verified that the each acquisition was performed in almost uniform weather conditions. Before the MB processing, the coherences have been compensated for the noise decorrelation by using standard procedures relying on the noise information distributed with the data [1]. Moreover, interferogram phase offsets variable with the baseline have been compensated relying on a bare soil patch and an external SRTM DEM [1].

Figure 1 shows a Fourier tomographic slice extracted at constant azimuth in the height-range plane. The mainlobe is located in correspondence of the SRTM DEM, and position and amplitude of the sidelobes agree with the height pointspread function. Due to the poor tomographic resolution, no other relevant information on the scattering can be extracted.

\subsection{Accurate DEM estimation}

Assuming model (1), the DEM height $z_{D}$ has been estimated over a subset of the whole image by using (2), and it is shown in Figure 2 for the HH channel. The decorrelation pattern has been modelled as $\rho\left(k_{z, k}\right)=\rho_{0}^{k_{z} / k_{z 0}}$, where $k_{z 0}=\left(\max k_{z}\right) / K$ and $0<\rho_{0}<1$. The parameter $\rho_{0}$ is the so-called spread, meaning that the lower $\rho_{0}$ (tending to 0 ), the more the backscattered power is spread around $z_{D}$. The estimated spread is shown in Figure 3 for the same area considered in Figure 2. From a visual comparison with a Google Earth image, it has been seen that the areas with high $\rho_{0}$ (close to 1 , almost no spectral spread) corresponds to pastures, while $\rho_{0}$ decreases in correspondence of forests, as it is

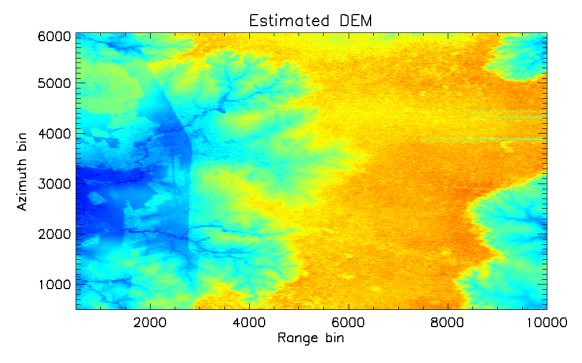

Fig. 2. Accurate estimate of a high resolution DEM over a range-azimuth subset. Color scale from blue $(0 \mathrm{~m})$ to red (250m).

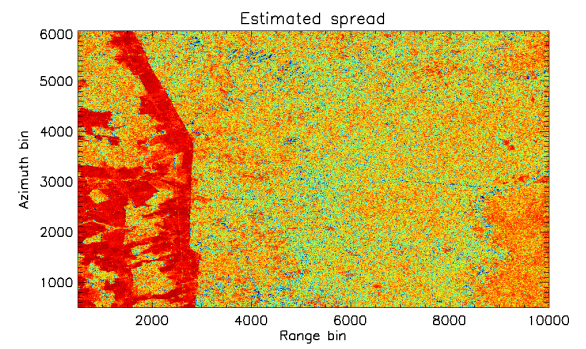

Fig. 3. Estimate of the coherence spread along the baseline. Color scale from blue (0.7) to red (1).

reasonable to expect. One can also consider the $-3 \mathrm{~dB}$ width of the spectrum corresponding to the chosen decorrelation pattern as an indication of penetration in the forest canopy. For the considered subset, it has been found a spectral width between a few meters and $15 \mathrm{~m}$.

As a first-cut experiment, the DEM of Figure 2 has been used to detect large trees by means of a local maximum filtering as suggested in [5], and a close-up image of the positions of the detected trees is reported in Figure 4. Although no direct validation was possible, the tree map looks reasonable as the detected trees are sparse and with distances in the order of magnitude of $100 \mathrm{~m}$ between themselves, as it is resonable to expect.

\subsection{Vertical structure}

First results are reported of full vertical structure estimation with the method proposed in Section 2.2. The constants $a$ and $b$ have been calculated as the ones resulting in the higher height difference between SMs.

Figures 5-6 show the tomographic slices estimated with the adaptive beamforming (Capon-based) of the separated SMs, classified into mechanisms at higher heights and at lower heights, respectively. For each cell, the presence of two scattering mechanism was considered only if the ratio between their polarimetric span was higher than $-10 \mathrm{~dB}$. When 2 SMs are present (more than $50 \%$ of the cells), their height difference resulted between a few meters and $20 \mathrm{~m}$. From 


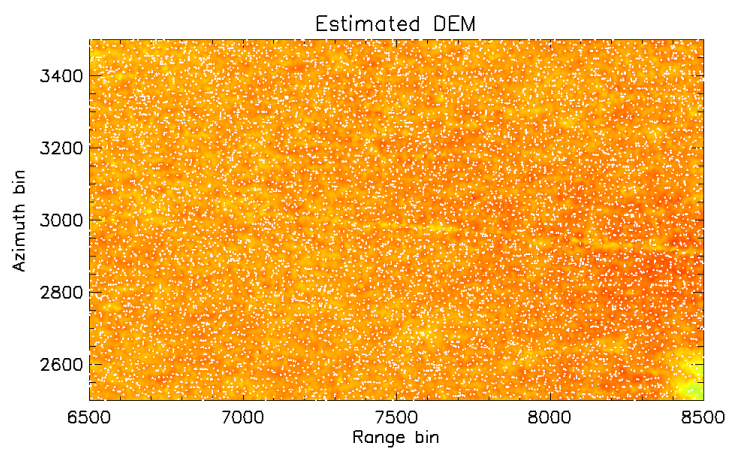

Fig. 4. White dots: detected large trees positions superimposed to the estimated DEM.

Figures 5-6 it is also visible a diversity of structure between different cells. To quantify this diversity, the model in (1) has been fitted to the coherences estimated for the two SM, and the spread $\rho_{0}$ estimated. It has been seen that different spreads correspond to different cells in the same SM (lower for bare soil and higher for forest canopy), and in general also for the two mechanisms in the same cell.

\section{DISCUSSION AND CONCLUSIONS}

In this paper, the possibility has been shown for the first time of estimating forest vertical structure from temporal decorrelation-free PolInSAR coherences provided by a spaceborne tandem system. Algorithmic solution have been presented for processing coherence vectors, thus further extending classical tomographic concepts. Experiments have been carried out by means of a TSX-TDX time series over the tropical forest of Tapajos.

This paper provided also some first results and insights about the information on forest vertical structure contained in $\mathrm{X}$-band data, following the experiments on assessing penetration and the performance in forest height estimation. It has been shown that MB PolInSAR data could be used for DEM estimation with high horizontal resolution. For instance, this kind of product could be used for mapping and monitoring large trees in tropical forest. It has also been shown that the full vertical structure can be retrieved with high vertical resolution and low ambiguities. In this case, the availability of different polarization channels is of key importance.

First 3-D experiments allow to conclude that X-band data are sensitive to forest structure, confirming the experimental evidence in [8] on a single-interferogram basis. Retaining the hypothesis of random volume, two scattering mechanisms could be separated with generally slightly different spectral characteristic. Future work will focus on the comparison between the estimated radar structure and ground and Lidar measurements, in order to further explore and validate the data information content.

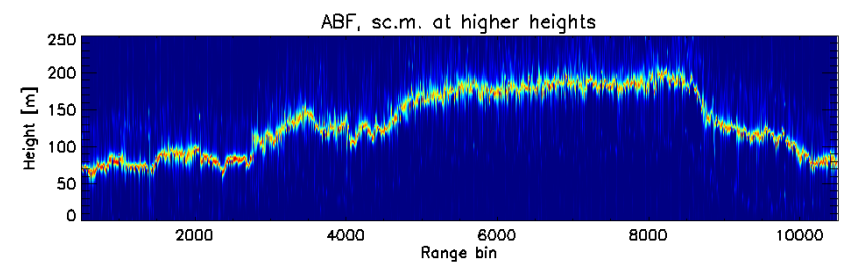

Fig. 5. ABF spectra at the same coordinates of Figure 1, higher scattering mechanism.

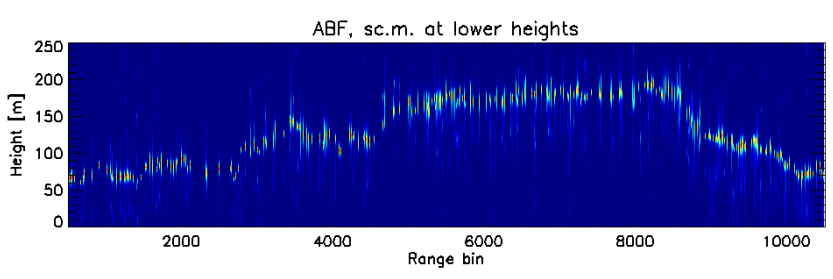

Fig. 6. ABF spectra at the same coordinates of Figure 1, lower scattering mechanism, after selection.

\section{REFERENCES}

[1] F. Kugler, at al., "Forest Characterisation by Means of TanDEM-X Pol-InSAR Data ," sumitted to IEEE Trans. on Geosci. and Rem. Sensing.

[2] G. Krieger, at al., "TanDEM-X: A Satellite Formation for High-Resolution SAR Interferometry," IEEE Trans. on Geosci. and Rem. Sensing, vol. 45, 2007.

[3] A. Toraño-Caicoya, at al., "Boreal Forest Biomass Classification With TanDEM-X," Proc. of ESA PolInSAR Workshop, Frascati, Italy, 2013.

[4] S. Cloude, "Polarization Coherence Tomography," Radio Science, vol. 41, 2006.

[5] T. Neef, at al., "Modeling Spatial Tree Patterns in the Tapajos Forest using Interferometric Height," Revista Brasileira de Cartografia, no. 57/01, 2005.

[6] F. Lombardini, at al., "Sector-Matched Interpolation for 3D SAR Correlative Interferometric Imaging," techn. rep., Edizioni ETS, Pisa, Italy, 2008.

[7] S. Tebaldini, "Algebraic Synthesis of Forest Scenarios From Multibaseline PolInSAR Data," IEEE Trans. on Geosci. and Rem. Sensing, vol. 47, 2009.

[8] F. De Zan, at al., "On Some Spectral Properties of TanDEM-X Interferograms Over Forested Areas," Geosci. and Rem. Sens. Letters, Vol. 10, 2013. 